\title{
Intrinsic flavor violation for massive neutrinos
}

\author{
C. C. Nishi* \\ Instituto de Física "Gleb Wataghin" \\ Universidade Estadual de Campinas, Unicamp \\ 13083-970, Campinas, SP, Brasil and \\ Instituto de Física Teórica, UNESP - São Paulo State University \\ Rua Pamplona, 145, 01405-900 - São Paulo, Brasil
}

\begin{abstract}
It is shown that intrinsic neutrino flavor violation invariably occurs when neutrinos are created within the SM augmented by the known massive neutrinos, with mixing and nondegenerate masses. The effects are very small but much greater than the naive estimate $\Delta m^{2} / E_{\nu}^{2}$ or the branching ratio of indirect flavor violating processes such as $\mu \rightarrow e \gamma$ within the SM. We specifically calculate the probability (branching ratio) of pion decay processes with flavor violation, such as $\pi \rightarrow \mu \bar{\nu}_{e}$, showing nonzero results.

PACS numbers: 14.60.Pq, 13.15.+g, 11.10.-z
\end{abstract}

*Electronic address: ccnishi@ifi.unicamp.br 


\section{INTRODUCTION}

After the confirmation that neutrinos are massive, nondegenerate and mix themselves, further investigations are being intensively carried out, experimentally as well as theoretically, to clarify the remaining mysteries about the neutrinos and the new physics they could be hiding [1]. One question that massive neutrinos immediately poses concerns the status of lepton number $(L)$ and family lepton numbers $\left(L_{e}, L_{\mu}, L_{\tau}\right)$ that were automatically conserved in the standard model (SM) without right-handed singlet neutrinos. We know from the successful observation of neutrino flavor oscillations that family lepton numbers are not conserved quantities due to the presence of the nondiagonal MNS mixing matrix. Total lepton number could be conserved at the classical level if neutrinos were Dirac fermions but that scenario does not explain the smallness of neutrino masses. The most natural way to explain tiny neutrino masses is the seesaw mechanism but, in this case, neutrinos are Majorana fermions in general. Although, approximate lepton number conservation can be achieved, guaranteeing small active neutrino masses, by assigning appropriate lepton numbers to heavy SM gauge singlets [2].

Indeed, it is exactly in the seesaw scenario that many interesting physics could be potentially observable. If the seesaw scale is relatively low, at the order of $\mathrm{TeV}$, effects such as the violation of unitarity of the MNS matrix [3] may be observable or the direct production of heavy seesaw particles [2, 4], including heavy neutrinos (type I or III) or heavy scalars (type II), might be possible. Non-standard interactions could also modify the standard oscillation formulas [5]. In such context, it is common to think that all consequences of the SM augmented by massive neutrinos have been investigated through. (An extensive analysis can be found in Ref.66.) Most of the direct consequences of massive neutrinos, with the exception of neutrino oscillations, are very difficult to be observed due to the tiny masses and mass differences: $\left|\Delta m_{12}^{2}\right| \approx 8 \times 10^{-5} \mathrm{eV}^{2}$ and $\left|\Delta m_{23}^{2}\right| \approx 2.3 \times 10^{-3} \mathrm{eV}^{2}[7]$. For example, the production of antineutrinos with negative helicity is possible in principle, because neutrinos are massive, but negligible in practice [8]. Despite such difficulties, an enormous experimental effort is being dispended to measure the absolute neutrino mass scale [9]. On the other hand, indirect effects allowed by massive neutrinos with mixing, such as the lepton flavor (LF) violating decay $\mu \rightarrow e \gamma$, are even strongly suppressed in the $\mathrm{SM}\left[\operatorname{Br}(\mu \rightarrow e \gamma)<10^{-50}\right]$ because of the tiny neutrino masses that enter the loops [10, 11]. Extensions of the SM, 
though, may lead in general to relatively large LF violating effects and certain conditions should be fulfilled for a natural suppression [12].

Contrary to usual expectations, we will show in this article that intrinsic neutrino flavor violation, hence lepton flavor violation, is possible in neutrino creation due solely to the known neutrino mass differences and nonzero mixing. More specifically, we will show that processes such as $\pi \rightarrow \mu \bar{\nu}_{e}$, are possible with a branching ratio much greater than loop induced processes such as $\mu \rightarrow e \gamma$. In fact, this effect should be correctly quantified before considering new physics contributions that could mimic the same effects [13, 14]. For instance, there were attempts to explain the LSND anomaly [15] from new physics interactions that violate lepton flavor [16]. For interactions that conserve total lepton number, however, conflicts with low energy phenomena can not be avoided [17]. Before the confirmation that neutrino oscillations were responsible for both deficits of neutrinos coming from the sun and the atmosphere, there were attempts to explain the deficit with non-standard interactions [18], even with massless neutrinos[19]. Indeed, it is important to distinguish the intrinsic lepton flavor violation effect calculated here from effects coming from interactions, extrinsic to the presence of neutrino masses, that violate lepton flavor and, perhaps, lepton number. Such interactions could give rise to effective operators with observable consequences in other low energy phenomena. An analogous distinction between direct and indirect CP violation is important to classify the CP violating effects involving the neutral $K$-mesons [20] that confirmed the CKM mechanism of CP violation in the SM [21].

The outline of the article is as follows: in Sec.II we apply the Wigner-Weisskopf approximation to treat the pion decay, considering the finite decay width. Section $\amalg$ contains the main results of neutrino flavor violation in pion decay and uses mainly Eqs. (10) and (30) from Sec.II. The ones only interested in the results may skip Sec.II. We discuss the results and some implications in Sec.IV] The appendices show some calculations that were omitted through the text and some useful material.

\section{WIGNER-WEISSKOPF APPROXIMATION IN PION DECAY}

Consider the pion decay $\pi^{-} \rightarrow l_{i}^{-}+\bar{\nu}_{j}, i=1,2\left(l_{1} \equiv e, l_{2} \equiv \mu\right)$ and $j=1,2,3$. The detailed description of this decay will be made by applying the Wigner-Weisskopf (WW) approximation method [22]. The WW method is essentially an improved method of second 
order time dependent perturbation theory which can describe the dynamics of decaying and decayed states at intermediate times (exponential behavior).

To calculate the decaying pion state at any time $t$, within the applicable approximation that only $l_{i} \bar{\nu}_{j}$ states appear as decay states, it suffices to discover the functions $\psi$ and $\chi$ in

$$
|\pi(t)\rangle_{\mathrm{ww}}=\int d^{3} \mathrm{p} \psi(\mathbf{p}, t) e^{-i E_{\pi} t}|\pi(\mathbf{p})\rangle+\sum_{i j} \int d^{3} \mathrm{q} d^{3} \mathrm{k} \chi_{i j}(\mathbf{q}, \mathbf{k} ; t) e^{-i\left(E_{l_{i}}+E_{\nu_{j}}\right) t}\left|l_{i}(\mathbf{q}) \nu_{j}(\mathbf{k})\right\rangle
$$

where the spin degrees of freedom are omitted and the states $\left\{|\pi(\mathbf{p})\rangle,\left|l_{i}(\mathbf{q}) \nu_{j}(\mathbf{k})\right\rangle\right\}, i, j=1,2$, refer to the free states, eigenstates of $H_{0}$, normalized as

$$
\begin{aligned}
\left\langle\pi\left(\mathbf{p}^{\prime}\right) \mid \pi(\mathbf{p})\right\rangle & =\delta^{3}\left(\mathbf{p}-\mathbf{p}^{\prime}\right) \\
\left\langle l_{i}\left(\mathbf{q}^{\prime}\right) \nu_{j}\left(\mathbf{k}^{\prime}\right) \mid l_{i}(\mathbf{q}) \nu_{j}(\mathbf{k})\right\rangle & =\delta^{3}\left(\mathbf{q}-\mathbf{q}^{\prime}\right) \delta^{3}\left(\mathbf{k}-\mathbf{k}^{\prime}\right) .
\end{aligned}
$$

The expansion (11) means we are restricted to the lowest order of perturbation theory.

The free Hamiltonian is characterized by the free energy of the states with physical masses

$$
\begin{aligned}
H_{0}|\pi(\mathbf{p})\rangle & =E_{\pi}(\mathbf{p})|\pi(\mathbf{p})\rangle \\
H_{0}\left|l_{i}(\mathbf{q}) \nu_{j}(\mathbf{k})\right\rangle & =\left(E_{l_{i}}(\mathbf{q})+E_{\nu_{j}}(\mathbf{k})\right)\left|l_{i}(\mathbf{q}) \nu_{j}(\mathbf{k})\right\rangle,
\end{aligned}
$$

where $E_{\alpha}(\mathbf{p})=\sqrt{\mathbf{p}^{2}+M_{\alpha}^{2}}\left(\alpha=\pi, l_{i}, \nu_{j}\right)$, and we will denote $M_{l_{i}} \equiv M_{i}$ and $M_{\nu_{j}} \equiv m_{j}$. The interaction Hamiltonian is given by

$$
V=-\int d^{3} \mathrm{x} \mathscr{L}_{F}(\mathbf{x})+\text { counter terms }
$$

where $\mathscr{L}_{F}$ is the Fermi interaction Lagrangian.

Considering the total Hamiltonian

$$
H=H_{0}+V \text {, }
$$

we can write a Schrödinger-like equation

$$
\begin{aligned}
\left(i \frac{d}{d t}-H_{0}\right)|\pi(t)\rangle_{\mathrm{ww}}= & \int d^{3} \mathrm{p} i \frac{\partial \psi(\mathbf{p}, t)}{\partial t} e^{-i E_{\pi} t}|\pi(\mathbf{p})\rangle \\
& +\sum_{i j} \int d^{3} \mathrm{q} d^{3} \mathrm{k} i \frac{\partial \chi_{i j}(\mathbf{q}, \mathbf{k} ; t)}{\partial t} e^{-i\left(E_{l_{i}}+E_{\nu_{j}}\right) t}\left|l_{i}(\mathbf{q}) \nu_{j}(\mathbf{k})\right\rangle \\
= & V|\Psi(t)\rangle
\end{aligned}
$$

Contraction with the appropriate states yields

$$
\begin{aligned}
i \frac{\partial}{\partial t} \psi(\mathbf{p}, t) & =\frac{\delta M^{2}}{2 E_{\pi}} \psi(\mathbf{p}, t)+\sum_{i j} \int d^{3} \mathrm{q} d^{3} \mathrm{k} \chi_{i j}(\mathbf{q}, \mathbf{k} ; t)\left\langle\pi(\mathbf{p})|V(t)| l_{i}(\mathbf{q}) \nu_{j}(\mathbf{k})\right\rangle, \\
i \frac{\partial}{\partial t} \chi_{i j}(\mathbf{q}, \mathbf{k} ; t) & =\int d^{3} \mathrm{p} \psi(\mathbf{p}, t)\left\langle l_{i}(\mathbf{q}) \nu_{j}(\mathbf{k})|V(t)| \pi(\mathbf{p})\right\rangle
\end{aligned}
$$


where $V(t)=e^{i H_{0} t} V e^{-i H_{0} t}$ and $\delta M^{2}$ is a counter term.

From the initial conditions

$$
\begin{aligned}
\psi(\mathbf{p}, 0) & =\psi(\mathbf{p}), \\
\chi_{i j}(\mathbf{q}, \mathbf{k} ; 0) & =0,
\end{aligned}
$$

we can formally solve

$$
\chi_{i j}(\mathbf{q}, \mathbf{k} ; t)=-i \int_{0}^{t} d t^{\prime} \int d^{3} \mathrm{p} \psi\left(\mathbf{p}, t^{\prime}\right)\left\langle l_{i}(\mathbf{q}) \nu_{j}(\mathbf{k})\left|V\left(t^{\prime}\right)\right| \pi(\mathbf{p})\right\rangle
$$

and obtain

$$
\begin{aligned}
\frac{\partial}{\partial t} \psi(\mathbf{p}, t)=-i \frac{\delta M^{2}}{2 E_{\pi}} \psi(\mathbf{p}, t)+\int d^{3} \mathrm{p}^{\prime} d^{3} \mathrm{q} d^{3} \mathrm{k} \int_{0}^{t} d t^{\prime}\left\langle\pi(\mathbf{p})|V(t)| l_{i}(\mathbf{q}) \nu_{j}(\mathbf{k})\right\rangle \\
\times\left\langle l_{i}(\mathbf{q}) \nu_{j}(\mathbf{k})\left|V\left(t^{\prime}\right)\right| \pi\left(\mathbf{p}^{\prime}\right)\right\rangle \psi\left(\mathbf{p}^{\prime}, t^{\prime}\right) .
\end{aligned}
$$

This is the key equation for the WW approximation.

Notice that only momentum conservation holds for the matrix elements, in particular,

$$
\left\langle l_{i}(\mathbf{q}) \nu_{j}(\mathbf{k})|V| \pi(\mathbf{p})\right\rangle=N_{i j}^{-1 / 2} \mathscr{M}_{i j} \delta^{3}(\mathbf{p}-\mathbf{q}-\mathbf{k})
$$

where $N_{i j}=(2 \pi)^{3} 2 E_{l_{i}}(\mathbf{q}) 2 E_{\nu_{j}}(\mathbf{k}) 2 E_{\pi}(\mathbf{p})$ and $\mathscr{M}_{i j} \equiv \mathscr{M}_{i j}(\mathbf{p}, \mathbf{q}, \mathbf{k})=\mathscr{M}\left(\pi^{-}(\mathbf{p}) \rightarrow l_{i}^{-}(\mathbf{q}) \bar{\nu}_{j}(\mathbf{k})\right)$. Replacing Eq. (15) into Eq. (14) yields

$$
\frac{\partial}{\partial t} \psi(\mathbf{p}, t)=-i \frac{\delta M^{2}}{2 E_{\pi}} \psi(\mathbf{p}, t)-\frac{1}{2 E_{\pi}(\mathbf{p})} \int_{0}^{t} d t^{\prime} \psi\left(\mathbf{p}, t-t^{\prime}\right) K\left(\mathbf{p}, t^{\prime}\right)
$$

where

$$
K\left(\mathbf{p}, t^{\prime}\right)=\frac{1}{(2 \pi)^{3}} \sum_{i j} \int \frac{d^{3} \mathrm{q}}{2 E_{l_{i}}} \frac{d^{3} \mathrm{k}}{2 E_{\nu_{j}}} e^{i \Delta E_{i j} t^{\prime}}\left|\mathscr{M}_{i j}\right|^{2} \delta^{3}(\mathbf{p}-\mathbf{q}-\mathbf{k}),
$$

where $\Delta E_{i j} \equiv E_{\pi}-E_{l_{i}}-E_{\nu_{j}}$ and the respective $\mathbf{p}, \mathbf{q}, \mathbf{k}$ dependence of $E_{\pi}, E_{l_{i}}, E_{\nu_{j}}$ is implicit. The expression in Eq. (17), however, does not provide a convergent integral since $\left|\mathscr{M}_{i j}\right|^{2}$ behaves as $\mathbf{k}^{2}$ for $\mathbf{q}=\mathbf{p}-\mathbf{k}$ and $|\mathbf{k}| \rightarrow \infty$. However, a cutoff function $f(\mathbf{p}, \mathbf{q}, \mathbf{k})$ multiplying $\mathscr{M}_{i j}$ is understood to regularize the expression. Such function can arise effectively from the pion form factor and vertex corrections in higher orders [23]. Such cutoff function is necessary to ensure the convergence of Eq. (17) and the production rate of $\pi(\mathbf{p}) \rightarrow l_{i}(\mathbf{q}) \bar{\nu}_{j}(\mathbf{k})$ to be more probable for the energy conserving states and do not grow indefinitely for high $|\mathbf{k}|$. We will assume that the cutoff function $f$ satisfies the properties 
(P1) the functional form of $f$ is broad for $E_{l_{i}}$ or $E_{\nu_{j}}$ and it varies very slowly for values close to the energy conserving values, in particular $f=1$ for $\Delta E_{i j}=0$.

(P2) the suppression of high momentum $|\mathbf{k}|$ or $|\mathbf{q}|$ (with $\mathbf{q}+\mathbf{k}$ fixed) occurs only significantly at an scale $\Lambda$ which satisfies $\Gamma \ll \Lambda \ll M_{\pi}^{2} / \Gamma$, where $\Gamma$ is the pion decay width.

Only these properties will be necessary for most of the calculations in this article. The inclusion of an explicit cutoff function will be considered in appendix $\mathrm{A}$ to justify the property (P2).

With the introduction of $f$ we can argue that the dominant contribution of $K(\mathbf{p}, t)$ is for $t \sim 0$, since Eq. (17) corresponds to a Fourier transform in $E_{\nu_{j}}$ and the integrand is a very broad function, which leads to a narrow function in time. We can then approximate Eq. (14) as

$$
\frac{\partial}{\partial t} \psi(\mathbf{p}, t) \approx-i \frac{\delta M^{2}}{2 E_{\pi}} \psi(\mathbf{p}, t)-\frac{1}{2 E_{\pi}(\mathbf{p})}\left[\int_{0}^{\infty} d t^{\prime} K\left(\mathbf{p}, t^{\prime}\right)\right] \psi(\mathbf{p}, t)
$$

The Eq. (18) corresponds to the WW approximation and it is valid for intermediate times, i.e., $t$ should be greater than the time width of $K(\mathbf{p}, t)$, since for such short time the original expression (16) can be significantly different. Within the WW approximation the expression inside the bracket in Eq. (18) gives

$$
\int_{0}^{\infty} d t^{\prime} K\left(\mathbf{p}, t^{\prime}\right)=\frac{i}{(2 \pi)^{3}} \sum_{i j} \int \frac{d^{3} \mathrm{q}}{2 E_{l_{i}}} \frac{d^{3} \mathrm{k}}{2 E_{\nu_{j}}} \frac{\left|f \mathscr{M}_{i j}\right|^{2}}{\Delta E_{i j}+i \epsilon} \delta^{3}(\mathbf{p}-\mathbf{q}-\mathbf{k}) .
$$

Using the relation

$$
\frac{1}{E \pm i \epsilon}=\mathcal{P} \frac{1}{E} \mp i \pi \delta(E)
$$

we obtain

$$
\begin{aligned}
& \text { Re Eq. (19) }=\frac{\pi}{(2 \pi)^{3}} \sum_{i j} \int \frac{d^{3} \mathrm{q}}{2 E_{l_{i}}} \frac{d^{3} \mathrm{k}}{2 E_{\nu_{j}}}\left|f \mathscr{M}_{i j}\right|^{2} \delta^{4}(\mathbf{p}-\mathbf{q}-\mathbf{k}), \\
& \operatorname{Im} \text { Eq. (19) }=\frac{1}{(2 \pi)^{3}} \sum_{i j} \mathcal{P} \int \frac{d^{3} \mathrm{q}}{2 E_{l_{i}}} \frac{d^{3} \mathrm{k}}{2 E_{\nu_{j}}} \frac{\left|f \mathscr{M}_{i j}\right|^{2}}{\Delta E_{i j}} \delta^{3}(\mathbf{p}-\mathbf{q}-\mathbf{k}) \text {. }
\end{aligned}
$$

Using the property (P1) of $f$ we can identify Eq. (21) as proportional to the pion decay rate at rest [23]

$$
\text { Re Eq. (19) }=M_{\pi} \Gamma \text {, }
$$

while Eq. (22) can be absorbed by the counterterm

$$
\text { Re Eq. (19) }=-\delta M^{2} \text {. }
$$


We can finally find the functions $\psi$ and $\chi$. Equation (18) gives

$$
\frac{\partial}{\partial t} \psi(\mathbf{p}, t)=-\frac{\Gamma}{2 \gamma} \psi(\mathbf{p}, t)
$$

which can be readily solved to give

$$
\psi(\mathbf{p}, t)=\psi(\mathbf{p}) e^{-\Gamma t / 2 \gamma}
$$

in accordance to the expected exponential decay law. The factor $\gamma=E_{\pi}(\mathbf{p}) / M_{\pi}$ accounts for the Lorentz dilatation of time. At the same time, the production wave function can be obtained from Eq. (10)

$$
\begin{aligned}
\chi_{i j}(\mathbf{q}, \mathbf{k} ; t) & =\left.\tilde{\chi}_{i j}(\mathbf{p}, \mathbf{q}, \mathbf{k} ; t) \psi(\mathbf{p})\right|_{\mathbf{p}=\mathbf{q}+\mathbf{k}} \\
\tilde{\chi}_{i j}(\mathbf{p}, \mathbf{q}, \mathbf{k} ; t) & \equiv\left[1-e^{-i\left(\Delta E_{i j}-i \Gamma / 2 \gamma\right) t}\right] N_{i j}^{-1 / 2} \frac{f \mathscr{M}_{i j}(\mathbf{p}, \mathbf{q}, \mathbf{k})}{\Delta E_{i j}-i \frac{\Gamma}{2 \gamma}} .
\end{aligned}
$$

Thus $\left|\chi_{i j}(\mathbf{q}, \mathbf{k} ; t)\right|^{2}$ is the production probability density.

From the conservation of probability at any time $t$, we must check if

$$
\int d^{3} \mathrm{p}|\psi(\mathbf{p}, t)|^{2}+\sum_{i j} \int d^{3} \mathrm{q} d^{3} \mathrm{k}\left|\chi_{i j}(\mathbf{q}, \mathbf{k} ; t)\right|^{2}=1 .
$$

The calculation is performed in appendix B. The important point is that Eq. (29) is satisfied if we neglect the terms that does not conserve energy in the squared amplitude $\left|\mathscr{M}_{i j}\right|^{2}$, i.e., the second term in

$$
\sum_{\text {spins }}\left|\mathscr{M}_{i j}\right|^{2}=\left|\mathscr{M}_{i j}^{\mathrm{EC}}\right|^{2}+\left|\delta \mathscr{M}_{i j}\right|^{2}
$$

where the upperscript EC stands for energy conservation. Notice that the usual energy conserving term $\left|\mathscr{M}_{i j}^{\mathrm{EC}}\right|^{2}$ is positive definite while $\left|\delta \mathscr{M}_{i j}\right|^{2}$ has no definite sign. The cutoff function $f$ is responsible for controlling such contributions. Therefore we retain only the energy conserving parts of $\left|\mathscr{M}_{i j}\right|^{2}$ further on.

For future use, we also define

$$
M_{\pi} \Gamma_{i j}=\frac{\pi}{(2 \pi)^{3}}\left|\mathscr{M}_{i j}^{\mathrm{EC}}\right|^{2} \int d \Omega_{k}\left[\left(\frac{k^{2}}{2 E_{l_{i}} 2 E_{\nu_{j}}}\left(\frac{d\left(E_{l_{i}}+E_{\nu_{j}}\right)}{d k}\right)^{-1}\right]_{\mathrm{EC}}{ }^{\prime}\right.
$$

and

$$
\Gamma_{i}=\sum_{j} \Gamma_{i j}
$$


The ratio $\Gamma_{i} / \Gamma$ corresponds to the branching ratio of the reaction $\pi \rightarrow l_{i}+\bar{\nu}$, independent of neutrino flavor, and it practically coincides with the usual branching ratio calculated with massless neutrinos, since $\sum_{j}\left|U_{i j}\right|^{2}=1$ and the kinematical contribution of neutrino masses are negligible. Obviously, $\sum_{i} \Gamma_{i}=\Gamma$.

As a last remark, we should emphasize that nowhere in this section the precise form of the interaction was used, except in the asymptotic behavior of $\left|\mathscr{M}_{i j}\right|^{2}$. Therefore, this approximation can be used in any two-body decay for which the interaction Hamiltonian is known, as long as a proper cutoff function is understood. The explicit amplitude $\mathscr{M}_{i j}$ and squared amplitude $\left|\mathscr{M}_{i j}\right|^{2}$ for pion decay are shown in appendix $\mathrm{C}$,

\section{NEUTRINO FLAVOR VIOLATION IN PION DECAY}

We begin by defining the two-particle states with definite flavor [24]

$$
\left|l_{\alpha}(\mathbf{q}) \bar{\nu}_{\beta}(\mathbf{k})\right\rangle \equiv \delta_{\alpha i} U_{\beta j}\left|l_{i}(\mathbf{q}) \bar{\nu}_{j}(\mathbf{k})\right\rangle
$$

The charged lepton states remain as mass eigenstates while the neutrino states are mixed through $U_{\beta j}$. We will see, in accordance to usual expectations, that pions decay mainly into the states $\left|l_{\alpha}(\mathbf{q}) \bar{\nu}_{\beta}(\mathbf{k})\right\rangle$ with $(\alpha, \beta)=(\mu, \mu)$. However, we will also see that there is a non-null probability of the pion to decay into the neutrino flavor violating states with $(\alpha, \beta)=(\mu, e)$ or $(\alpha, \beta)=(e, \mu)$. For that purpose, we want to ultimately calculate the probability

$$
\mathcal{P}_{l_{\alpha} \nu_{\beta}}(t)=\int d^{3} \mathbf{q} \int d^{3} \mathbf{k} \sum_{\text {spins }}\left|\left\langle l_{\alpha}(\mathbf{q}) \bar{\nu}_{\beta}(\mathbf{k}) \mid \pi(t)\right\rangle_{\mathrm{ww}}\right|^{2} .
$$

Using $\chi_{i j}$ in Eq. (10), when $t \gg 1 / \Gamma$, we can rewrite Eq. (34) as

$$
\mathcal{P}_{l_{\alpha} \nu_{\beta}}(t)=\int d^{3} \mathbf{p}|\psi(\mathbf{p})|^{2} \int d^{3} \mathbf{k} \sum_{\text {spins }}\left|\sum_{j} U_{\alpha j} e^{-i E_{\nu_{j}} t} U_{j \beta}^{\dagger} F_{\alpha j}\right|_{\mathbf{q}=\mathbf{p}-\mathbf{k}}^{2},
$$

where

$$
U_{\alpha j} F_{\alpha j}(\mathbf{p}, \mathbf{q}, \mathbf{k}) \equiv N_{\alpha j}^{-1 / 2} \frac{f \mathscr{M}_{\alpha j}(\mathbf{p}, \mathbf{q}, \mathbf{k})}{\Delta E_{\alpha j}-i \frac{\Gamma}{2 \gamma}} .
$$

We see the exponential $e^{-i E_{\nu_{j}} t}$ is responsible for the neutrino oscillation phenomenon. In fact, if we neglect the neutrino mass $m_{j}$ in every term of Eq. (35), except in the exponential, we get

$$
\mathcal{P}_{l_{\alpha} \nu_{\beta}}(t)=\int d^{3} \mathbf{p}|\psi(\mathbf{p})|^{2} \int d^{3} \mathbf{k} \mathcal{P}_{\nu_{\alpha} \nu_{\beta}}(t)\left|F_{\alpha}\right|^{2}
$$


where $F_{\alpha}=\left(F_{\alpha j}\right)_{m_{j} \rightarrow 0}$. Notice the usual oscillation probability,

$$
\mathcal{P}_{\nu_{\alpha} \nu_{\beta}}(t)=\left|\sum_{j} U_{\alpha j} e^{-i E_{\nu_{j}}(\mathbf{k}) t} U_{j \beta}^{\dagger}\right|^{2}
$$

factors out from the creation probability of $l_{\alpha} \bar{\nu},\left|F_{\alpha}\right|^{2}$, for massless neutrinos. Such factorization is what allows the definition of the state Eq. (33) as a flavor state, since

$$
\mathcal{P}_{l_{\alpha} \nu_{\beta}}(t) \approx \delta_{\alpha \beta} \frac{\Gamma_{\alpha}}{\Gamma}
$$

for $1 / \Gamma \ll t \ll L_{\mathrm{osc}}$, where $L_{\mathrm{osc}}$ is the typical flavor oscillation length (period). Therefore, the antineutrino flavor state $U_{\alpha j}\left|\bar{\nu}_{j}\right\rangle$ is only created jointly with the charged lepton $l_{\alpha}[25,26]$. Notice Eq. (39) correctly coincides with the branching ratio of the decay $\pi \rightarrow l_{\alpha} \bar{\nu}$. Neutrinos, however, are not strictly massless and we may have initial flavor violation because different neutrino masses contribute differently to each channel $\pi \rightarrow l_{i}+\bar{\nu}_{j}[25]$. We will focus on initial flavor violation and denote the interval of time satisfying $1 / \Gamma \ll t \ll L_{\text {osc }}$ by $t=0$.

We can make the flavor violating contributions explicit by rewriting the term inside the square modulus in Eq. (35) as

$$
\sum_{j=1}^{3} U_{\alpha j} U_{\beta j}^{*} F_{\alpha j}=\delta_{\alpha \beta} F_{\alpha 1}+\sum_{j=2}^{3} U_{\alpha j} U_{\beta j}^{*} \Delta F_{\alpha j}
$$

where $\Delta F_{\alpha j} \equiv F_{\alpha j}-F_{\alpha 1}$. Thus the square modulus becomes

$$
\left|\sum_{j=1}^{3} U_{\alpha j} U_{\beta j}^{*} F_{\alpha j}\right|^{2}=\delta_{\alpha \beta}\left|F_{\alpha 1}\right|^{2}+\delta_{\alpha \beta} 2 \operatorname{Re}\left[F_{\alpha 1}^{*} \sum_{j=2}^{3} U_{\alpha j} U_{\beta j}^{*} \Delta F_{\alpha j}\right]+\left|\sum_{j=2}^{3} U_{\alpha j} U_{\beta j}^{*} \Delta F_{\alpha j}\right|^{2} .
$$

We recognize that only the last term of Eq. (41) is flavor non-diagonal. The second term, which is flavor diagonal, is estimated in appendix $\mathrm{E}$ and shown to be much smaller than the flavor violating contribution.

Specializing to $\alpha \neq \beta$, under the approximation of $U_{\alpha 3} U_{\beta 3}^{*} \approx 0$ (which is valid if $\alpha=e$ or $\beta=e$ ), the initial creation probability yields

$$
\mathcal{P}_{l_{\alpha} \nu_{\beta}}(0)=\int d^{3} \mathbf{p}|\psi(\mathbf{p})|^{2} \int d^{3} \mathbf{k}\left|U_{\alpha 2} U_{\beta 2}^{*}\right|^{2}\left|\Delta F_{\alpha 2}\right|^{2} .
$$

For the two family parametrization, we have $\left|U_{\alpha 2} U_{\beta 2}^{*}\right|^{2}=\frac{1}{4} \sin ^{2} 2 \theta$, thus indicating that this phenomenon is indeed mixing dependent.

To analyze the most dominant contribution to Eq. (42), we recall that a general function $g(x)$ can be expanded

$$
g(x+a)-g(x-a) \approx g^{\prime}(x) 2 a,
$$


for small enough $a$. Moreover, if $g(x)=\prod_{i=1}^{n} g_{i}(x)$, the relative difference can be written

$$
\frac{g(x+a)-g(x-a)}{g(x)} \approx 2 a \sum_{i}^{n} \frac{g_{i}^{\prime}(x)}{g_{i}(x)} .
$$

Taking $x$ to be $m_{2}^{2}=\overline{m^{2}}+\frac{1}{2} \Delta m^{2}$ and $a=\frac{1}{2} \Delta m^{2}=\frac{1}{2}\left(m_{2}^{2}-m_{1}^{2}\right)$ we can estimate the different contributions that compose $F_{\alpha 2}$ :

$(\delta 1) g=E_{\nu_{2}}^{-1 / 2}: \quad a \frac{g^{\prime}}{g}=-\frac{\Delta m^{2}}{4 \bar{E}_{\nu}^{2}}$,

$(\delta 2) g=\left(\Delta E_{\alpha 2}-i \Gamma / 2 \gamma\right)^{-1}: \quad a \frac{g^{\prime}}{g}=i \frac{\Delta m^{2}}{2 \bar{E}_{\nu} \Gamma} \gamma$,

$(\delta 3) g=|\mathbf{k}|_{2}^{\mathrm{EC}}: \quad a \frac{g^{\prime}}{g} \sim \frac{\Delta m^{2}}{2 \mathbf{k}^{2}}$,

$(\delta 4) \frac{\left|\tilde{\mathscr{M}}_{\alpha 2}^{\mathrm{EC}}\right|^{2}-\left|\tilde{\mathscr{M}}_{\alpha 1}^{\mathrm{EC}}\right|^{2}}{\left|\tilde{\mathscr{M}}_{\alpha 2}^{\mathrm{EC}}\right|_{m_{2} \rightarrow 0}^{2}} \approx \frac{\Delta m^{2}}{2 M_{\alpha}^{2}}\left(\frac{M_{\pi}^{2}+2 M_{\alpha}^{2}}{M_{\pi}^{2}-M_{\alpha}^{2}}\right)$.

We are assuming the energy conserving values $\left(\Delta E_{i j} \approx 0\right)$, which is an excellent approximation considering $\bar{E}_{\nu},|\mathbf{k}|$ are essentially the same either if we compute it using $m_{2}^{2}$ or $m_{1}^{2}$. Conventionally we will take the simple average $\overline{m^{2}}=\frac{1}{2}\left(m_{1}^{2}+m_{2}^{2}\right)$. In particular, in $(\delta 3),|\mathbf{k}|_{2}^{\text {EC }}$ denotes the momentum of neutrino $\nu_{2}$, assuming energy conservation and $\mathbf{p} \approx 0$ : $|\mathbf{k}|_{2}^{\mathrm{EC}}=\sqrt{E_{\nu_{2}}^{2}-m_{2}^{2}}$, where $E_{\nu_{2}}=\left(M_{\pi}^{2}-M_{\alpha}^{2}+m_{2}^{2}\right) /\left(2 M_{\pi}\right)$. We also note that $\Gamma \gg \Delta m^{2} / 2 \bar{E}_{\nu}$ is satisfied recalling $\Gamma=2.53 \times 10^{-8} \mathrm{eV}[27]$ and $\Delta m^{2} / 2 \bar{E}_{\nu} \sim \frac{1}{6} \times 10^{-7} \mathrm{eV}^{\frac{\Delta m^{2}}{1 \mathrm{eV}^{2}}}$, where $\Delta m^{2}$ is either $\left|\Delta m_{12}^{2}\right| \sim 0.8 \times 10^{-4}$ or $\left|\Delta m_{23}^{2}\right| \sim 2.5 \times 10^{-3}[7]$. This condition is necessary to have coherent flavor neutrino creation [25]. From $\Gamma \ll \bar{E}_{\nu},|\mathbf{k}|^{\mathrm{EC}}$, it is also clear that among the different contributions $(\delta n)$, the dominant contribution is given by $(\delta 2)$. A through analysis of the difference between the amplitudes $\mathscr{M}_{\alpha j}$, estimated in $(\delta 4)$, is shown in appendix C. Therefore we can neglect all differences due to $\Delta m^{2}$ in $F_{\alpha j}$ except in the terms $\left(\Delta E_{\alpha j}-i \Gamma / 2 \gamma\right)^{-1}$ and obtain

$$
\begin{aligned}
\left|\Delta F_{\alpha 2}\right|^{2} & \approx \frac{\left|\mathscr{M}_{\alpha+}^{\mathrm{EC}}\right|^{2}}{N_{\alpha+}}\left|\frac{1}{\Delta E_{\alpha 2}-i \frac{\Gamma}{2 \gamma}}-\frac{1}{\Delta E_{\alpha 1}-i \frac{\Gamma}{2 \gamma}}\right|^{2} \\
& \approx \frac{\left|\mathscr{M}_{\alpha+}^{\mathrm{EC}}\right|^{2}}{N_{\alpha+}}\left(\frac{\Delta m^{2}}{2 E_{\nu}}\right)^{2} \frac{1}{\left[\left(\Delta E_{\alpha+}\right)^{2}+\frac{\Gamma^{2}}{4 \gamma^{2}}\right]^{2}}
\end{aligned}
$$

where the subscript + means we assume $m_{2}^{2}=m_{1}^{2}=\overline{m^{2}}$, as well as in $E_{\nu}=\sqrt{\mathbf{k}^{2}+\overline{m^{2}}}$. It is also implicit that $\left|\mathscr{M}_{\alpha+}^{\mathrm{EC}}\right|^{2}$ refers to $\left|\mathscr{M}_{\alpha j}^{\mathrm{EC}}\right|^{2}$ with $m_{j}^{2} \rightarrow \overline{m^{2}}$ and without the mixing matrix 
element $\left|U_{\alpha j}\right|^{2}$ [see Eq. (36) $)$. Notice we are already assuming $\mathbf{k}^{2} \gg \overline{m^{2}}$, otherwise the term inside parenthesis should be kept as $E_{\nu_{2}}(\mathbf{k})-E_{\nu_{1}}(\mathbf{k})$. Although the $|\mathbf{k}| \rightarrow 0$ limit of such term in Eq. (46) is well defined and gives $\frac{1}{2} \Delta m^{2} / \sqrt{\overline{m^{2}}} \sim \Delta m=m_{2}-m_{1}$. In practice, for realistic $|\mathbf{k}|$, we could assume massless neutrinos for these terms.

The flavor violating creation probability in Eq. (42) can be calculated in analogy to Eq. (B4), using Eq. (31), which gives

$$
\mathcal{P}_{l_{\alpha} \nu_{\beta}}(0) \approx \frac{1}{2} \sin ^{2} 2 \theta \frac{\Gamma_{\alpha}}{\Gamma}\left(\frac{\Delta m^{2}}{2 E_{\nu} \Gamma}\right)_{\mathrm{EC}}^{2},
$$

where the two family parametrization, $\left|U_{\alpha 2} U_{\beta 2}^{*}\right|^{2}=\frac{1}{4} \sin ^{2} 2 \theta$, was employed and $\mathbf{p} \approx 0$ (pion at rest) was considered by adjusting $\psi(\mathbf{p})$. The following integral was also necessary,

$$
\int_{-\infty}^{\infty} d \lambda \frac{1}{\left[\lambda^{2}+\frac{\Gamma^{2}}{4 \gamma^{2}}\right]^{2}}=\frac{2 \pi}{\Gamma}\left(\frac{2 \gamma^{2}}{\Gamma^{2}}\right)
$$

One can recognize the term inside parenthesis in Eqs. (46) and (48) as the additional contribution that appears in Eq. (47).

Let us estimate some specific flavor violation probabilities (branching ratios):

$$
\frac{\mathcal{P}_{\mu \nu_{e}}(0)}{\sin ^{2} 2 \theta_{12}} \sim 10^{-9}, \frac{\mathcal{P}_{e \nu_{\mu}}(0)}{\sin ^{2} 2 \theta_{12}} \sim 3 \times 10^{-15} \frac{\Gamma_{e}}{\Gamma}, \frac{\mathcal{P}_{\mu \nu_{\tau}}(0)}{\sin ^{2} 2 \theta_{23}} \sim 10^{-6} .
$$

To compute the last value in Eq. (49), we considered $\left|\Delta m_{13}^{2}\right| \approx\left|\Delta m_{23}^{2}\right| \gg\left|\Delta m_{12}^{2}\right|$.

\section{DISCUSSIONS}

The important point of this detailed calculation is that lepton flavor violation should necessarily occur when neutrinos are created because it is unlikely that the expression in Eq. (42) would cancel exactly. It is also important to emphasize that neutrinos should be detected as flavor states, as defined (approximately) in Eq. (33), to observe the flavor violation effects. The coherent creation of neutrino flavor states is indeed guaranteed from the observations of neutrino oscillations. When neutrinos are not explicitly detected, their effects can be computed from an incoherent sum of the contributions of each neutrino mass eigenstate [6], as in the intended direct measurements of absolute neutrino mass. Extensive investigations in such context, were first reported in Ref. 6 . On the other hand, if mass eigenstates were created and detected incoherently, flavor violating effects would be analogous to flavor changing processes for quarks, at tree level, without the explicit appearance of the $\Delta m^{2}$ dependence. 
The neutrino flavor violation effects reported here are, in general, very small but relatively larger than what would be expected from a naive estimate $\Delta m^{2} / E_{\nu}^{2}$ (a similar result is indeed obtained in Refs. 28 and 29) because of the presence of the finite decay width $\Gamma$, which is very small for pions. We could define, differently from Eq. (33), that the neutrinos created jointly with the charged lepton $\alpha$ is $\nu_{\alpha}$ by definition [26]. However, the difference between such definition [Eq. (3.16) of Ref.[26a] and the usual definition in Eq. (33) carries the factor $\Delta m^{2} / E_{\nu}^{2}$ and it is negligible compared to the factor we have calculated in Eq. (47). Thus the effect calculated in Eq. (47) is dominant, even if we distinguish the neutrinos created from different sources [26]. In fact, intrinsic neutrino flavor violation effects can not be large because otherwise there would be no coherent creation of neutrino flavor states and there would be no flavor oscillation [25, 26, 30]. Of course, this analysis is modified if there are genuine non-standard interactions [13].

Previous calculations of intrinsic neutrino flavor violation [26, 28, 29] did not explicitly considered the contribution of the finite decay width of the parent particle and either neglected the effect [26] or considered it unphysical [28, 29]. The arguments of Ref. 28 is based on a formalism that uses a unitarily inequivalent vacuum that guarantees initial neutrino flavor conservation [28] but also implies slightly different oscillation formulas [31]. Instead, the intrinsic flavor violation effect calculated in Eq. (47) should be regarded as a genuine physical consequence of massive neutrinos with mixing and it contradicts neither the weak Hamiltonian as stated in Ref.29 nor any experimental observations. The qualitative occurrence of intrinsic neutrino flavor violation, that in the context of flavor oscillations could be called initial flavor violation, could be anticipated in more phenomenological calculations of flavor oscillation probabilities considering scalar [32] or fermionic [33, 34] wave packets but its magnitude could not be determined without the full consideration of the interaction responsible for neutrino creation.

The expression in Eq. (41) reminds the $\Delta S=2$ contribution from box diagrams in $K^{0}$ $\bar{K}^{0}$ mixing (see, e.g., Ref.23, p. 235). Such contribution is suppressed by the GIM mechanism [35] because it involves the sum of the contributions of quarks $u, c$ and $t$ in the loop. Equation (41), however, is not loop suppressed and, differently for quarks, the mixing angles are large. These facts explain the relatively large effect calculated in Eq. (47), despite tiny neutrino mass differences. In fact, the effect is much larger than loop suppressed effects such as the lepton flavor violating decay $\mu \rightarrow e \gamma$ in the SM. Although, in models beyond the SM 
such as the MSSM, such effects can be larger than the current experimental limit [11].

Despite the arbitrariness of the cutoff function $f$, the expression in Eq. (42) is finite independently of the presence of that function. This feature shows the robustness of the calculation as the cutoff scale $\Lambda$ may be chosen from a wide range without affecting the results. A expression very similar to Eq. (47) was estimated in an unrealistic exactly solvable QFT model of Lee-type in Ref.25, also showing that the intrinsic neutrino flavor violation effects calculated here bear some universality independently of the particular interaction in question.

The further inclusion of radiative corrections to the formalism developed in Sec.I does not seem to be straightforward. The corrections have to be included without spoiling the conservation of probability of Eq. (29). It is also possible that deviations from the exponential decay law would emerge from such corrections or from an approximation scheme distinct from the WW approximation. Deviations from exponential behavior are indeed expected for very short or very long times from the unitary evolution of quantum mechanics [36]. A brief connection with perturbative QFT is also shown in appendix E. The inclusion of finite widths in perturbative QFT is interesting in its own right because it mixes up different orders in perturbation theory and special care is necessary in gauge theories to keep track of gauge invariance [37]. Obviously, to fully specify the dominant cutoff scale $\Lambda$, radiative corrections should be explicitly considered. The study of the renormalization procedure also needs careful analysis. In this respect, it should be emphasized that the necessity of the cutoff function $f$ is not related to the nonrenormalizability of the Fermi interaction. The same asymptotic behavior $\left(\left|\mathscr{M}_{i j}\right| \sim \mathbf{k}^{2}\right)$ would require a cutoff function if instead we adopted a Yukawa-type interaction which is renormalizable. In the context of neutrino propagation and oscillation, the inclusion of finite widths was also considered in Refs. [38, 39] at lowest order.

Another possible application of the formalism developed in Sec.II concerns the study of the effects of the finite width to the effective size of the decaying particles. The roles played by the finite width and the intrinsic momentum uncertainty, encoded here in the wave function $\psi(\mathbf{p})$, are not clear but they are crucial to the occurrence of neutrino oscillations, a phenomenon that requires quantum coherence. As it is well known, a small uncertainty in the spatial localization of the neutrinos are necessary to the observation of neutrino oscillations [30]. With such formalism, the quantum entaglement can be also studied, differently 
of the static Lee-type model [25]. The extension to three-body decays should be also pursued since most of the decays with neutrino creation, such as the beta decay or $\mu \rightarrow e \bar{\nu}_{e} \nu_{\mu}$, have three decay particles. In that respect, it is important to notice that the kinematics of a three-body decay is very different from a two-body decay that emits monoenergetic particles when the parent particle is at rest.

To summarize, intrinsic neutrino flavor violation should occur when neutrino flavor states are created. The effect is the consequence of the slightly different creation amplitudes, functions of different neutrino masses, that have to be summed coherently. The smallness of the effect explains why neutrino flavor is an approximately well defined concept in the SM and it is directly related to the smallness of the neutrino mass differences. At the same time, small mass splittings allow the coherent creation of neutrino flavor states that is required for the phenomenon of neutrino flavor oscillations. The observation of the latter enabled the recent progress in understanding some of the fundamental properties of neutrinos.

\section{APPENDIX A: THE CUTOFF FUNCTION $f$}

We will show here that the contribution to Eq. (B2) coming from the second term of Eq. (30) is negligible for a cutoff function $f$ that obeys the properties (P1) and (P2). We will adopt the particular function

$$
|f(\mathbf{p}, \mathbf{q}, \mathbf{k})|^{2}=\frac{\Lambda^{2}}{\left(\Delta E_{i j}\right)^{2}+\Lambda^{2}} .
$$

Close to the energy conserving values the contribution of $\left|\delta \mathscr{M}_{i j}\right|^{2}$ is negligible compared to $\left|\mathscr{M}_{i j}^{\mathrm{EC}}\right|^{2}$ as we can see in Eq. ([C8). To analyze the contribution of $\left|\delta \mathscr{M}_{i j}\right|^{2}$ for $|\mathbf{k}| \gg \Gamma$, we rewrite Eq. (C7) as

$$
\left|\delta \mathscr{M}_{i j}\right|^{2}=\left(\Delta E_{i j}\right)^{2} A_{2}+\Delta E_{i j} A_{1},
$$

and note that the coefficients $A_{2}, A_{1}$ are bounded functions of $|\mathbf{k}|$. More specifically, $E_{l_{i}}(\mathbf{p}-$ $\mathbf{k})-E_{\nu_{j}}(\mathbf{k}) \approx E_{l_{i}}(\mathbf{p}-\mathbf{k})-|\mathbf{k}|$ is a monotonically decreasing function bounded by $E_{l_{i}}(\mathbf{p})$ and $-|\mathbf{p}| \cos \theta$. We also notice that the term inside parenthesis in Eq. (B33) is bounded as

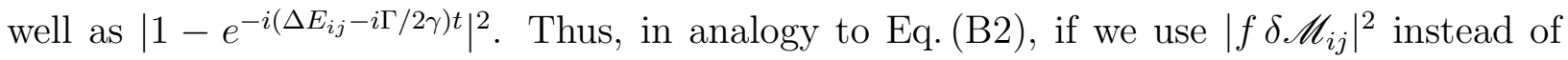
$\left|\mathscr{M}_{i j}^{\mathrm{EC}}\right|^{2}$, inside the integral in $d k$, we recognize we have to compare

$$
\int_{E_{l_{i}}(\mathbf{p})-E_{\pi}}^{\infty} d \lambda \frac{\left|f \delta \tilde{\mathscr{M}}_{i j}\right|^{2}}{\lambda^{2}+\frac{\Gamma^{2}}{4 \gamma^{2}}}
$$


with

$$
\int_{E_{l_{i}}(\mathbf{p})-E_{\pi}}^{\infty} d \lambda \frac{\left|\tilde{\mathscr{M}}_{i j}^{\mathrm{EC}}\right|^{2}}{\lambda^{2}+\frac{\Gamma^{2}}{4 \gamma^{2}}},
$$

where $\lambda=-\Delta E_{i j}$ and we are neglecting the neutrino masses. Taking only the contribution of $A_{2}$ in Eq. (A2), the ratio between Eqs. (A3) and (A4) is

$$
\begin{aligned}
R & \lesssim \frac{\left|A_{2}\right|_{\max } \Lambda^{2}}{\left|\tilde{\mathscr{M}}_{i j}^{\mathrm{EC}}\right|^{2}}\left(\frac{2 \pi \gamma}{\Gamma}\right)^{-1} \int_{-\infty}^{\infty} \frac{\lambda^{2}}{\left[\lambda^{2}+\frac{\Gamma^{2}}{4 \gamma^{2}}\right]\left[\lambda^{2}+\Lambda^{2}\right]} \\
& \sim \frac{\Lambda \Gamma}{2\left(M_{\pi}^{2}-M_{i}^{2}\right)} \ll 1,
\end{aligned}
$$

assuming $\mathbf{p} \approx 0$ and $(\mathrm{P} 2)$ is valid. The contribution coming from $A_{1}$ is much smaller.

\section{APPENDIX B: CALCULATION OF EQ. (29)}

The second term of Eq. (29) can be rewritten as

$$
\int d^{3} \mathrm{q} d^{3} \mathrm{k}\left|\chi_{i j}(\mathbf{q}, \mathbf{k} ; t)\right|^{2}=\int d^{3} \mathrm{p}|\psi(\mathbf{p})|^{2} \int d^{3} \mathrm{k}\left|\tilde{\chi}_{i j}(\mathbf{p}, \mathbf{p}-\mathbf{k}, \mathbf{k} ; t)\right|^{2},
$$

where we used the change of variable $\mathbf{q} \rightarrow \mathbf{p}=\mathbf{q}+\mathbf{k}$ and the sum over spins is implicit.

We can calculate, using (P1), the second integral of Eq. (B1) assuming that the contribution of the second piece of Eq. (30) is negligible and noticing that the squared amplitude, summed over spins, after imposing energy conservation, is a function only of the masses:

$$
\begin{aligned}
\int d^{3} \mathrm{k}\left|\tilde{\chi}_{i j}\right|^{2} & =\frac{\left|\mathscr{M}_{i j}^{\mathrm{EC}}\right|^{2}}{2 E_{\pi}(2 \pi)^{3}} \operatorname{Re} \int d \Omega_{k} \int_{0}^{\infty} d k\left(\frac{k^{2}}{2 E_{l_{i}} 2 E_{\nu_{j}}}\right) \frac{\left[1+e^{-\Gamma t / \gamma}-2 e^{-i\left(\Delta E_{i j}-i \frac{\Gamma}{2 \gamma}\right) t}\right]}{\left(\Delta E_{i j}\right)^{2}+\frac{\Gamma^{2}}{4 \gamma^{2}}} \\
& \approx \frac{\left|\mathscr{M}_{i j}^{\mathrm{EC}}\right|^{2}}{2 E_{\pi}(2 \pi)^{3}} \operatorname{Re} \int d \Omega_{k}\left(\frac{k^{2}}{2 E_{l_{i}} 2 E_{\nu_{j}}} \frac{d k}{d \lambda}\right)_{\mathrm{EC}} \int_{-\infty}^{\infty} d \lambda \frac{\left[1+e^{-\Gamma t / \gamma}-2 e^{i\left(\lambda-E_{\pi}+i \frac{\Gamma}{2 \gamma}\right) t}\right]}{\left(\lambda-E_{\pi}\right)^{2}+\frac{\Gamma^{2}}{4 \gamma^{2}}}( \\
& =\frac{\left|\mathscr{M}_{i j}^{\mathrm{EC}}\right|^{2}}{M_{\pi}(2 \pi)^{3}} \operatorname{Re} \int d \Omega_{k}\left(\frac{k^{2}}{2 E_{l_{i}} 2 E_{\nu_{j}}} \frac{d k}{d \lambda}\right)_{\mathrm{EC}} \frac{\pi}{\Gamma}\left[1-e^{-\Gamma t}\right]
\end{aligned}
$$

recalling that $\gamma=E_{\pi} / M_{\pi}$. In Eq. (B33), the change of variables $|\mathbf{k}| \rightarrow \lambda=E_{\nu_{j}}+E_{l_{i}}$ was used and the lower end of the integral was extended to $-\infty$, considering $M_{\pi}-M_{i}-m_{j} \gg \Gamma$.

Comparing Eq. (B4) with Eqs. (21) and (31), after using $\Gamma=\sum_{i j} \Gamma_{i j}$, we see Eq. (29) is satisfied. 


\section{APPENDIX C: PION DECAY}

The effective Fermi interaction lagrangian is

$$
\mathscr{L}_{F}=-2 \sqrt{2} G_{F}\left(\bar{l}_{i}(x) \gamma^{\mu} L U_{i j} \nu_{j}(x)\right) J_{\mu}(x)+\text { h.c. },
$$

where $L=\frac{1}{2}\left(1-\gamma_{5}\right),\left\{U_{i j}\right\}$ denotes the MNS matrix while $J_{\mu}$ is the hadronic current that in the case of pion decay reads

$$
J_{\mu}=V_{u d} \bar{u}_{L} \gamma_{\mu} d_{L}
$$

Using $\mathscr{L}_{F}$ we can calculate $\mathscr{M}_{i j}$ in Eq. (15):

$$
\mathscr{M}_{i j}=i C U_{i j} \bar{u}_{i}(\mathbf{q}) \not p L v_{j}(\mathbf{k}) \equiv i C U_{i j} \tilde{\mathscr{M}}_{i j}(\mathbf{p}, \mathbf{q}, \mathbf{k})
$$

where $C \equiv 2 F_{\pi} G_{F} V_{u d}$. We have used the chiral current relation [23]

$$
\left\langle 0\left|\bar{u}(x) \gamma_{\mu} \gamma_{5} d(x)\right| \pi^{-}(\mathbf{p})\right\rangle=-i \sqrt{2} F_{\pi} \frac{p_{\mu}}{\sqrt{2 E_{\pi}(\mathbf{p})}} \frac{e^{-i p . x}}{(2 \pi)^{3 / 2}},
$$

where $F_{\pi} \approx 92 \mathrm{MeV}$ is the pion decay constant. It is important to keep in mind that Eq. (C3) should be calculated without assuming energy conservation. In that case, the squared amplitude is

$$
\sum_{\text {spins }}\left|\tilde{\mathscr{M}}_{i j}(\mathbf{p}, \mathbf{q}, \mathbf{k})\right|=4\left(p \cdot q_{i}\right)\left(p \cdot k_{j}\right)-2\left(q_{i} \cdot k_{j}\right) p^{2}
$$

where $p^{\mu} \equiv\left(E_{\pi}(\mathbf{p}), \mathbf{p}\right), q_{i}^{\mu} \equiv\left(E_{l_{i}}(\mathbf{q}), \mathbf{q}\right)$ and $k_{j}^{\mu} \equiv\left(E_{\nu_{j}}(\mathbf{k}), \mathbf{k}\right)$. If we consider energy conservation, we get the usual

$$
\sum_{\text {spins }}\left|\tilde{\mathscr{M}}_{i j}(\mathbf{p}, \mathbf{q}, \mathbf{k})\right|_{\mathrm{EC}}=\left|\tilde{\mathscr{M}}_{i j}^{\mathrm{EC}}\right|=M_{i}^{2}\left(M_{\pi}^{2}-M_{i}^{2}\right)+m_{j}^{2}\left(M_{\pi}^{2}+2 M_{i}^{2}-m_{j}^{2}\right)
$$

without neglecting the neutrino masses. The remaining part of Eq. (C5) that does not conserve energy can be calculated by using $p=q+k+\delta p$, in four-vector notation, where $\delta p \equiv\left(\Delta E_{i j}, \mathbf{0}\right):$

$$
\left|\delta \mathscr{M}_{i j}\right|^{2}=\left(\Delta E_{i j}\right)^{2}\left[\mathbf{p}^{2}-\left(E_{l_{i}}-E_{\nu_{j}}\right)^{2}\right]-\left(M_{i}^{2}-m_{j}^{2}\right) 2 \Delta E_{i j}\left(E_{l_{i}}-E_{\nu_{j}}\right) .
$$

It is important to estimate

$$
\frac{\left|\delta \mathscr{M}_{i j}\right|^{2}}{\left|\mathscr{M}_{i j}^{\mathrm{EC}}\right|^{2}} \approx-\frac{M_{i}^{2}}{M_{\pi}^{2}-M_{i}^{2}}\left(\frac{2 \Gamma}{M_{\pi}}+\frac{\Gamma^{2}}{M_{\pi}^{2}}\right),
$$


considering $\Delta E_{i j} \sim \Gamma, \mathbf{p} \approx 0$, and the energy conserving values for the rest of the terms. Numerically Eq. (요 $)$ is dominated by $\Gamma / M_{\pi} \sim 10^{-16}$ which is negligible and it supports why we neglected the contribution of the terms $\left|\delta \mathscr{M}_{i j}\right|^{2}$ when computing the flavor violation probability in Eq. (47).

We can also calculate

$$
\sum_{\text {spins }} \tilde{\mathscr{M}}_{i j}(\mathbf{p}, \mathbf{q}, \mathbf{k}) \tilde{\mathscr{M}}_{i j^{\prime}}^{*}(\mathbf{p}, \mathbf{q}, \mathbf{k})=4\left(q_{i} \cdot p\right)\left(p \cdot\langle k\rangle_{j j^{\prime}}\right)-2 p^{2}\left(q_{i} \cdot\langle k\rangle_{j j^{\prime}}\right)
$$

where $\langle k\rangle_{j j^{\prime}}$ is given by Eq. (D2). To calculate Eq. ([C9), we made use of the completeness relation in Eq. (D1). Furthermore, the mixed squared amplitude in Eq. (C9) can be decomposed, in analogy to Eq. (301), as

$$
\sum_{\text {spins }} \tilde{\mathscr{M}}_{i j}(\mathbf{p}, \mathbf{q}, \mathbf{k}) \tilde{\mathscr{M}}_{i j^{\prime}}^{*}(\mathbf{p}, \mathbf{q}, \mathbf{k})=\left|\tilde{\mathscr{M}}_{i, j j^{\prime}}^{\mathrm{EC}}\right|^{2}+\left|\delta \tilde{\mathscr{M}}_{i, j j^{\prime}}\right|^{2}
$$

Energy conservation (EC) assumes the neutrino four-momentum is $\langle k\rangle_{j j^{\prime}}$. Since the mass associated to $\langle k\rangle_{12}$, for example, is $\sqrt{\langle k\rangle_{12}^{2}}=\sqrt{m_{1} m_{2}}$, i.e., the geometrical average, we can also show that

$$
\left|\tilde{\mathscr{M}}_{i 1}^{\mathrm{EC}}\right|^{2}<\left|\tilde{\mathscr{M}}_{i, 12}^{\mathrm{EC}}\right|^{2}<\left|\tilde{\mathscr{M}}_{i 2}^{\mathrm{EC}}\right|^{2}
$$

for $m_{1}<m_{2}$. Equation (C11) confirms that the contribution due to neutrino mass differences in the amplitudes $\mathscr{M}_{i j}$ can be indeed neglected in comparison to the contribution containing $\Gamma$, i.e., $(\delta 2)$, when computing Eq. (47).

\section{APPENDIX D: COMPLETENESS RELATIONS FOR SPINORS WITH DIFFER-} ENT MASSES

To compute Eq. (35) exactly, it is necessary to calculate mixed squared amplitudes such as $\sum_{\text {spin }} \mathscr{M}_{i 1} \mathscr{M}_{i 2}^{*}$, where the subscripts 1 and 2 denote spinors involving different masses, $m_{1}$ and $m_{2}$. We are interested, however, in calculating the sum over spins using a common basis for the spin directions for the spinors $v_{\nu_{1}}(\mathbf{k})$ and $v_{\nu_{2}}(\mathbf{k})$. (Depending on the parametrization adopted $\left.\bar{v}_{\nu_{1}}(\mathbf{k}, r) v_{\nu_{2}}(\mathbf{k}, s) \neq \delta_{r s}\right)$ The only basis where that is possible is their common helicity basis. In that basis we have, with helicity $h$,

$$
\sum_{h} v_{\nu_{1}}(\mathbf{k}, h) \bar{v}_{\nu_{2}}(\mathbf{k}, h)=\langle\not k\rangle_{21}-\gamma^{0} \Delta \not k_{21},
$$


where

$$
\begin{aligned}
\langle k\rangle_{21} & \equiv \sqrt{m_{1} m_{2}}(\cosh \bar{\xi}, \hat{\mathbf{k}} \sinh \bar{\xi}) \\
\Delta k_{21} & \equiv \sqrt{m_{1} m_{2}}(\cosh \Delta \xi, \hat{\mathbf{k}} \sinh \Delta \xi)
\end{aligned}
$$

with $\bar{\xi}=\frac{1}{2}\left(\xi_{1}+\xi_{2}\right)$ and $\Delta \xi=\xi_{2}-\xi_{1}$. The usual hyperbolic parametrization is employed, i.e., $k_{j}=m_{j}\left(\cosh \xi_{i}, \hat{\mathbf{k}} \sinh \xi_{i}\right)$, with the additional constraint $m_{1} \sinh \xi_{1}=m_{2} \sinh \xi_{2}=|\mathbf{k}|$. Notice that Eq. (D1) reduces to the usual $\not k-m$ when $m_{1}=m_{2}=m$.

To calculate Eq. (D1) we made use of the parametrization

$$
v_{\nu_{j}}(\mathbf{k}, h)=\frac{m_{j}-\not k_{j}}{\sqrt{m_{j}+E_{j}}} v_{0}(\mathbf{k}, h)
$$

where $E_{j}=\left(k_{j}\right)_{0}$, and the completeness relation

$$
\sum_{h= \pm} v_{0}(\mathbf{k}, h) v_{0}^{\dagger}(\mathbf{k}, h)=\frac{1}{2}\left(1-\gamma^{0}\right) .
$$

\section{APPENDIX E: FLAVOR CONSERVING EFFECTS}

Let us estimate the effect of the second term of Eq. (41) which is flavor diagonal. Comparing to Eqs. (46) and (47), it is possible that it could be relatively large, of the order of $\Delta m^{2} / E_{\nu} \Gamma$. However, we can calculate

$$
2 \operatorname{Re} F_{\alpha 1}^{*} \Delta F_{\alpha 2} \approx \frac{\left|\mathscr{M}_{\alpha+}^{\mathrm{EC}}\right|^{2}}{N_{\alpha+}} \frac{\Delta m^{2}}{2 E_{\nu}} \frac{2 \Delta E_{\alpha+}}{\left[\left(\Delta E_{\alpha+}\right)^{2}+\frac{\Gamma^{2}}{4 \gamma^{2}}\right]^{2}} .
$$

After integration in $\lambda=-\Delta E_{\alpha+}$, the effect is non-null only because of the lower integration limit, $\lambda_{0}=m_{j}+E_{l_{\alpha}}(\mathbf{p})-E_{\pi}(\mathbf{p}) \approx-M_{\pi}+M_{\alpha}$, is finite. One can see the contribution will be proportional to

$$
\left(\frac{2 \pi \gamma}{\Gamma}\right)^{-1} \frac{\Delta m^{2}}{2 E_{\nu}} \int_{\lambda_{0}}^{\infty} d \lambda \frac{2 \lambda}{\left[\lambda^{2}+\frac{\Gamma^{2}}{4 \gamma^{2}}\right]^{2}} \approx \frac{\Delta m^{2}}{2 E_{\nu} \Gamma} \frac{1}{2 \pi} \frac{\Gamma^{2}}{\lambda_{0}^{2}},
$$

which is, in general, much smaller than $\left(\frac{\Delta m^{2}}{2 E_{\nu} \Gamma}\right)^{2}$.

\section{APPENDIX F: CONNECTION WITH PERTURBATIVE QUANTUM FIELD}

\section{THEORY}

The treatment of unstable states in pertubative Quantum Field Theory (QFT) is of considerable interest since the majority of particles studied at high energies, including the 
ones we call elementary, are unstable and observed as resonances. We will briefly show the connection between the formalism developed in Sec. (III) with perturbative QFT.

Although the asymptotic "in" and "out" states can not be defined for an unstable state, we can still calculate

$$
\begin{aligned}
\psi(\mathbf{p}, t) & ={ }_{0}\left\langle\pi(\mathbf{p})|U(t, 0)| \pi_{\psi}\right\rangle_{0}, \\
\chi_{i j}(\mathbf{q}, \mathbf{k} ; t) & ={ }_{0}\left\langle l_{i}(\mathbf{q}), \bar{\nu}_{j}(\mathbf{k})|U(t, 0)| \pi_{\psi}\right\rangle_{0},
\end{aligned}
$$

where

$$
\left|\pi_{\psi}\right\rangle_{0}=\int d^{3} \mathbf{p} \psi(\mathbf{p})|\pi(\mathbf{p})\rangle_{0}
$$

and

$$
U\left(t, t^{\prime}\right)=T \exp \left[-i \int_{-t^{\prime}}^{t} V(t)\right]
$$

with $T$ being the time ordered product. Recall that the $S$ matrix is given by $U(\infty,-\infty)$.

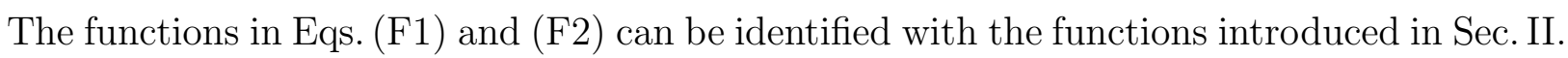
In particular, they obey the initial conditions of Eqs. (11) and (12). They also obey

$$
\begin{aligned}
i \frac{d}{d t} \psi(\mathbf{p}, t) & ={ }_{0}\left\langle\pi(\mathbf{p})|V(t) U(t, 0)| \pi_{\psi}\right\rangle_{0}, \\
i \frac{d}{d t} \chi_{i j}(\mathbf{q}, \mathbf{k} ; t) & ={ }_{0}\left\langle l_{i}(\mathbf{q}), \bar{\nu}_{j}(\mathbf{k})|V(t) U(t, 0)| \pi_{\psi}\right\rangle_{0} .
\end{aligned}
$$

In particular, if the completeness relation in Fock space could be truncated by $\mathbb{1}=|\pi\rangle\langle\pi|+$ $\sum_{i j}\left|l_{i} \bar{\nu}_{j}\right\rangle\left\langle l_{i} \bar{\nu}_{j}\right|$, we recover Eqs. (91) and (10).

\section{ACKNOWLEDGMENTS}

This work was supported by Fundação de Amparo à Pesquisa do Estado de São Paulo (Fapesp). The author would like to thank Prof. Orlando L. G. Peres for pointing out Ref. [13] and Prof. C. O. Escobar for Ref. [36].

[1] A. Y. Smirnov, "Neutrino physics: Open theoretical questions," Int. J. Mod. Phys. A 19 (2004) 1180 arXiv:hep-ph/0311259].

[2] J. Kersten and A. Y. Smirnov, "Right-Handed Neutrinos at LHC and the Mechanism of Neutrino Mass Generation,” Phys. Rev. D 76 (2007) 073005 arXiv:0705.3221 [hep-ph]]. 
[3] Z. z. Xing and S. Zhou, "Cosmic Neutrino Flavor Democracy and Unitarity Violation at Neutrino Telescopes," Phys. Lett. B 666 (2008) 166 [arXiv:0804.3512 [hep-ph]].

[4] W. Chao, Z. G. Si, Z. z. Xing and S. Zhou, "Correlative signatures of heavy Majorana neutrinos and doubly-charged Higgs bosons at the Large Hadron Collider," arXiv:0804.1265 [hep-ph].

[5] J. Kopp, M. Lindner, T. Ota and J. Sato, "Non-standard neutrino interactions in reactor and superbeam experiments," Phys. Rev. D 77 (2008) 013007 [arXiv:0708.0152 [hep-ph]]; M. Lindner, "Impact of non-standard interactions on neutrino physics," talk given at Workshop on the origin of P, CP and T violation, ICTP, Trieste, July 2-5, 2008.

[6] R. E. Shrock, "New Tests For, And Bounds On, Neutrino Masses And Lepton Mixing," Phys. Lett. B 96 (1980) 159; R. E. Shrock, "General Theory Of Weak Leptonic And Semileptonic Decays. 1. Leptonic Pseudoscalar Meson Decays, With Associated Tests For, And Bounds On, Neutrino Masses And Lepton Mixing," Phys. Rev. D 24 (1981) 1232.

[7] A. Strumia and F. Vissani, "Neutrino masses and mixings and ...," arXiv:hep-ph/0606054.

[8] C. C. Nishi, "Absolute neutrino mass from helicity measurements," arXiv:0805.2597 [hep-ph], to appear in Mod. Phys. Lett. A.

[9] C. Giunti, "Absolute neutrino masses," Acta Phys. Polon. B 36 (2005) 3215 arXiv:hep-ph/0511131.

[10] S. M. Bilenky and C. Giunti, "Lepton numbers in the framework of neutrino mixing," Int. J. Mod. Phys. A 16 (2001) 3931 arXiv:hep-ph/0102320.

[11] J. A. Casas and A. Ibarra, "Oscillating neutrinos and $\mu \rightarrow e \gamma$," Nucl. Phys. B 618 (2001) 171 arXiv:hep-ph/0103065.

[12] B. W. Lee and R. E. Shrock, "Natural Suppression Of Symmetry Violation In Gauge Theories: Muon - Lepton And Electron Lepton Number Nonconservation," Phys. Rev. D 16, 1444 (1977); A. Blum and A. Merle, "General Conditions for Lepton Flavor Violation at Tree- and 1-Loop Level," Phys. Rev. D 77, 076005 (2008) arXiv:0709.3294 [hep-ph]].

[13] Y. Grossman, "Nonstandard Neutrino Interactions And Neutrino Oscillation Experiments," Phys. Lett. B 359 (1995) 141 arXiv:hep-ph/9507344.

[14] L. M. Johnson and D. W. McKay, "Revising neutrino oscillation parameter space with direct flavor-changing interactions," Phys. Rev. D 61 (2000) 113007 arXiv:hep-ph/9909355; "Fitting direct interaction pieces into neutrino puzzles," Phys. Lett. B 433 (1998) 355 arXiv:hep-ph/9805311. 
[15] C. Athanassopoulos et al. [LSND Collaboration], Phys. Rev. C 54 (1996) 2685 arXiv:nucl-ex/9605001; Phys. Rev. Lett. 77 (1996) 3082 arXiv:nucl-ex/9605003; A. Aguilar et al. [LSND Collaboration], Phys. Rev. D 64, 112007 (2001) arXiv:hep-ex/0104049].

[16] S. Bergmann, H. V. Klapdor-Kleingrothaus and H. Pas, "Lepton number violation interactions and their effects on neutrino oscillation experiments," Phys. Rev. D 62 (2000) 113002 arXiv:hep-ph/0004048; K. S. Babu and S. Pakvasa, "Lepton number violating muon decay and the LSND neutrino anomaly," arXiv:hep-ph/0204236.

[17] S. Bergmann and Y. Grossman, "Can lepton flavor violating interactions explain the LSND results?," Phys. Rev. D 59 (1999) 093005 arXiv:hep-ph/9809524].

[18] S. Bergmann, Y. Grossman and D. M. Pierce, "Can lepton flavor violating interactions explain the atmospheric neutrino problem?," Phys. Rev. D 61 (2000) 053005 arXiv:hep-ph/9909390].

[19] M. M. Guzzo, A. Masiero and S. T. Petcov, "On the MSW effect with massless neutrinos and no mixing in the vacuum," Phys. Lett. B 260 (1991) 154; S. Bergmann, M. M. Guzzo, P. C. de Holanda, P. I. Krastev and H. Nunokawa, "Status of the solution to the solar neutrino problem based on non-standard neutrino interactions," Phys. Rev. D 62 (2000) 073001 arXiv:hep-ph/0004049.

[20] G. Buchalla, "CP violation in K and B decays," Talk given at 6th Conference on the Intersections of Particle and Nuclear Physics (CIPANP 97), Big Sky, MT, 27 May - 2 Jun 1997. In *Big Sky 1997, Intersections between particle and nuclear physics* 49-66 arXiv:hep-ph/9707545; D. London, "CP Violation in the B System: Measuring New-Physics Parameters," Int. J. Mod. Phys. A19 (2004) 5463. arXiv:hep-ph/0405241.

[21] M. Kobayashi and T. Maskawa, "CP Violation In The Renormalizable Theory Of Weak Interaction," Prog. Theor. Phys. 49 (1973) 652.

[22] V. Weisskopf and E. P. Wigner, "Calculation Of The Natural Brightness Of Spectral Lines On The Basis Of Dirac's Theory," Z. Phys. 63 (1930) 54. See also C. Cohen-Tannoudji, B. Diu and F. Laloë, Quantum Mechanics, vol 2, Complement $\mathrm{D}_{\mathrm{XIII}}$.

[23] J. F. Donoghue, E. Golowich, and B. R. Holstein, Dynamics of the Standard Model (Cambridge University Press, 1994).

[24] Recall that neutrino flavor states $\left|\nu_{\alpha}\right\rangle$ are superpositions of mass eigenstates $\left|\nu_{j}\right\rangle$ with coefficients $U_{\alpha j}^{*}$ while antineutrino flavor states $\left|\bar{\nu}_{\alpha}\right\rangle$ have coefficients $U_{\alpha j}[26]$.

[25] C. C. Nishi and M. M. Guzzo, "Flavor mixing in a Lee-type model," Phys. Rev. D 78 (2008) 
033008 [arXiv:0803.1422 [hep-ph]].

[26] C. Giunti, "Theory of neutrino oscillations," arXiv:hep-ph/0409230. Talk given at 16th Conference on High Energy Physics (IFAE 2004), Turin, Italy, 14-16 Apr 2004. Published in Turin 2004, High energy physics, 427-438; "Flavor neutrinos states," arXiv:hep-ph/0402217.

[27] S. Eidelman, et al. (Particle Data Group), Phys. Lett. B592, 1 (2004).

[28] M. Blasone, A. Capolupo, C. R. Ji and G. Vitiello, "Short Time Limit of Mixing Formalism in Quantum Field Theory," arXiv:hep-ph/0611106;

[29] Y. F. Li and Q. Y. Liu, "A paradox on quantum field theory of neutrino mixing and oscillations," JHEP 0610 (2006) 048 arXiv:hep-ph/0604069.

[30] B. Kayser, "On The Quantum Mechanics Of Neutrino Oscillation," Phys. Rev. D 24 (1981) 110.

[31] M. Blasone and G. Vitiello, "Quantum field theory of fermion mixing," Ann. Phys. 244, 283 (1995) [Erratum-ibid. 249, 363 (1996)] arXiv:hep-ph/9501263.

[32] S. De Leo, C. C. Nishi and P. P. Rotelli, "Wave packets and quantum oscillations," Int. J. Mod. Phys. A 19 (2004) 677.

[33] A. E. Bernardini and S. De Leo, "Dirac spinors and flavor oscillations," Eur. Phys. J. C 37, 471 (2004) arXiv:hep-ph/0411153; "Flavor and chiral oscillations with Dirac wave packets," Phys. Rev. D 71, 076008 (2005).

[34] C. C. Nishi, "First quantized approaches to neutrino oscillations and second quantization," Phys. Rev. D 73 (2006) 053013 arXiv:hep-ph/0506109].

[35] S. L. Glashow, J. Iliopoulos and L. Maiani, "Weak Interactions with Lepton-Hadron Symmetry," Phys. Rev. D 2 (1970) 1285.

[36] L. A. Khalfin, "Unconditional tests of fundamental discrete symmetries CP, T, CPT in rigorous quantum dynamics beyond the approximate Lee-Oehme-Yang theory,” Found. Phys. 27 (1997) 1549; C. B. Chiu and E. C. G. Sudarshan, "Decay and evolution of the neutral kaon," Phys. Rev. D 42 (1990) 3712; L. Fonda, G. C. Ghirardi and A. Rimini, Rept. Prog. Phys. 41 (1978) 587.

[37] E. N. Argyres et al., "Stable calculations for unstable particles: Restoring gauge invariance," Phys. Lett. B 358 (1995) 339 arXiv:hep-ph/9507216; A. Aeppli, G. J. van Oldenborgh and D. Wyler, "Unstable particles in one loop calculations," Nucl. Phys. B 428 (1994) 126 arXiv:hep-ph/9312212. 
[38] W. Grimus, P. Stockinger and S. Mohanty, "The field-theoretical approach to coherence in neutrino oscillations," Phys. Rev. D 59 (1998) 013011 arXiv:hep-ph/9807442; "Neutrino oscillations and the effect of the finite lifetime of the neutrino source," Phys. Rev. D $6 \mathbf{1}$ (1999) 033001 arXiv:hep-ph/9904285].

[39] M. Beuthe, "Oscillations of neutrinos and mesons in quantum field theory," Phys. Rept. 375, 105 (2003) arXiv:hep-ph/0109119]; consult the references therein for original contributions. 